\title{
OPENING SPEECH: 2007 INTERNATIONAL SYMPOSIUM ON COSMOLOGY AND PARTICLE ASTROPHYSICS
}

\author{
W-Y. PAUCHY HWANG \\ The Leung Research Center for Cosmology and Particle Physics, \\ Institute of Astrophysics, and Department of Physics, \\ National Taiwan University, Taipei 106, Taiwan \\ wyhwang@phys.ntu.edu.tw
}

I have several reasons to welcome you to this unique international conference.

First of all, the term "Cosmology" is becoming truly the experimental science in the last decade (not too long ago). The emerging experimental science is basically the cross-disciplinary field between physics and astronomy. Before that, in fact, cosmology was purely the theological or theoretical speculations.

As human being, we are proud to be closer to the truth, that is what we are striving for. A lot of people argue that Cosmology can be viewed as an ultimate science of the mankind.

Second reason for this conference to be rather unique is that the Nobel Foundation decided, in October (now November - a little bit more than a month apart), to celebrate the cosmology this year, by giving the Nobel Prize to John Mather and George Smoot (shown in the LeCosPA Dedication Ceremony as Fig. 1).

In terms of the time span between the Nobel prize (given to Mather and Smoot, a month ago) and the celebration symposium (any conference), this conference sets the record in my memory. Of course, it just happened that way and no one, perhaps except the God, can plan this sort of things beforehand. Likewise, no one would anticipate that on one day Cosmology could become a truly experimental science we are truly lucky for that.

In the International Year of Physics (which marked the centennial memorial of Einstein's three most important papers), another Nobel laureate C. N. Yang lectured and published the paper "Einstein's Impact on Theoretical Physics in the 21st Century". He said, "....It led to the discipline of modern cosmology which is destined to become one of the important scientific fields of the 21st century." Cited from AAPPS Bulletin, Vol. 15, No. 1, February 2005.

As shown in the LeCosPA Dedication Ceremony as Fig. 2, the anisotropy, at the level of $10^{-5}$, of the $3^{\circ}$ Cosmic Microwave Backgrounds (CMB) as measured by 
G. Smoot et al. Also shown in the LeCosPA Dedication Ceremony as Fig. 3, the major milestones of CMB Cosmology are displayed - from COBE (in which Mather and Smoot are key players), to WMAP (in 2003 \& 2006), and to Planck Surveyor (to be launched in late 2008). So, it's clear that we are at one of the most important moments in the history of the humankind.

Of course, scientists know all about this. In 17 April 2002, U.S. Academy of Sciences issued the draft of the "white" paper: "Connecting Quarks with the Cosmos: Eleven Science Questions for the New Century." Eventually published by a book, of which the cover is shown in Fig. 1.

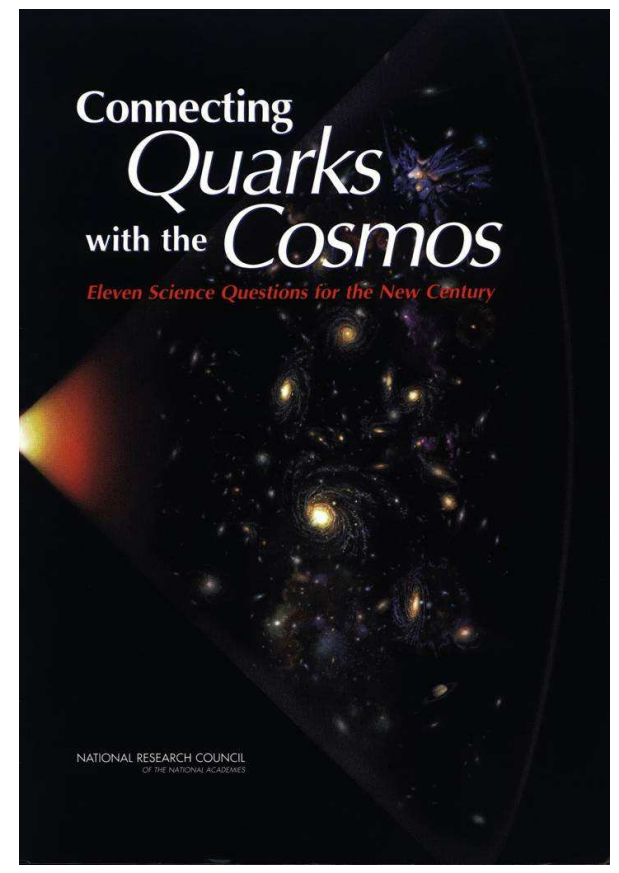

Fig. 1.

Connecting Quarks with the Cosmos: Eleven Science Questions for the New Century

Q1: What is the dark matter?

Q2: What is the nature of the dark energy?

Q3: How did the universe begin?

Q4: Did Einstein have the last word on gravity?

Q5: What are the masses of the neutrinos, and how have they shaped the evolution of the universe?

Q6: How do cosmic accelerators work and what are they accelerating?

Q7: Are protons unstable? 
Q8: Are there new states of matter at exceedingly high density and temperature? Q9: Are there additional spacetime dimensions?

Q10: How were the elements from iron to uranium made?

Q11: Is a new theory of matter and light needed at the highest energies?

Let me try to elaborate briefly these questions. First of all, these are experimental questions; that is, the answers are obtained by future experiments. Q1-Q3 relate to Cosmology nowadays - the composition of the dark matter and dark energy and what is the detail of the inflation, before the hot big bang. Q4 addresses the ultimate correctness of Einstein equation. And so on.

Our endeavors in the beginning of the twenty-first century in the fields of particle/nuclear physics and astrophysics will have remarkable implications on the ultimate science of the mankind, i.e., cosmology. We are indeed at an exciting period of the human history.

My famous biophysicist friend, Suney Chan, remarked (and I like to quote): "The "Large-Scale Universe" remains one of the unsolved mysteries of basic physical sciences. Knowledge of the cosmos is fundamental to our understanding of time, space, matter, energy, the formation of the elements, and the creation and evolution of life. All of us are interested in knowing about the origins of how we came into being, namely, the beginning of "our beginning", and where we are all going. It would be enormously exciting to make progress in this important area of science.

Modern biology helps us to look within us, but cosmology is destined to educate us on the origins of our psyche and what lies beyond."

I wish to extend my warmest welcome to you to Taiwan, especially when this is the first visit. Taiwan's name "Formosa" means the "beautiful island" although not much beauty left today because of these descendants.

Nevertheless, the hospitality should be there no matter what. Confucius once said, "What a Happiness it is to Have Friends who Come from Afar!!"

\section{Acknowledgments}

Supports and sponsorships from the following institutions are gratefully acknowledged:

Research Center on Cosmology and Particle Astrophysics, Nat'l Taiwan University Theoretical Institute for Advanced Research in Astrophysics (TIARA) Institute for Astronomy and Astrophysics, Academia Sinica (ASIAA)

National Science Council, Taiwan

Center for Theoretical Sciences (North), Taiwan

Asia Pacific Center for Theoretical Physics, Korea

KIPAC, SLAC, Stanford University

RESCEU, University of Tokyo 